\title{
A Singapore perspective on the use of a short course of chemothromboprophylaxis in patients who underwent total knee arthroplasty
}

\author{
Mun Hon Low ${ }^{1}$, MBBs, Mmed, Seng Jin $\underline{Y e o}^{1}$, FRCSE, Pak Lin $\underline{C h i n}^{1}$, FrCSE, Shi Lu Chia ${ }^{1}$, FrCSE,
} Ngai Nung $\underline{L O}^{1}$, FRCSE, Keng Jin $\underline{\text { Tay }}^{1}$, FRCSE

INTRODUCTION There is considerable controversy regarding the best method to prevent venous thromboembolism. In 2008, the American College of Chest Physicians (ACCP) published specific guidelines recommending the use of low-molecular-weight heparin or warfarin, and a target international normalised ratio of 2.0-3.0 for a duration of at least 7-10 days, after elective knee arthroplasties. Many orthopaedic surgeons believe that these recommendations are biased toward reducing deep venous thrombosis (DVT), but neglect the implicated possibility of a higher incidence of wound complications. In order to enable an objective evaluation of the fit of the ACCP recommendations to the needs of our local cohort of patients, we aimed to look at the incidence of DVT in our local population.

METHODS This study was a prospective observational study involving existing local patients in Singapore General Hospital, Singapore, who underwent total knee arthroplasty (TKA) and were on a short course of chemothromboprophylaxis (< 7 days) after the operation. The incidence of DVT in patients was evaluated using DVT imaging 4-6 days after the operation and at one month after the operation.

RESULTS In our study cohort, the prevalence of DVT during the period between postoperative Days 4 and 6 was $12 \%$ ( $11 \%$ were distal DVT and $1 \%$ was proximal DVT). Only $9 \%$ of the patients had DVT one month after the operation. Using chi-square analysis, we found that there was no significant increase in the number of DVT and pulmonary embolism cases 4-6 days and 1 month after the operation $(p>0.05)$.

CONCLUSION Contrary to the ACCP guidelines, a short course of chemothromboprophylaxis post TKA, lasting no more than 7 days, is safe and adequate in the low-risk Asian population.

Keywords: Asians, deep venous thrombosis, deep venous thrombosis incidence, deep venous thrombosis prophylaxis, total knee arthroplasty

\section{INTRODUCTION}

The latest American College of Chest Physicians (ACCP) guidelines recommend the use of antithrombotic prophylaxis (e.g. low-molecular-weight heparin or warfarin, rivoroxaban, fondaparinux and apixaban) for a duration of at least 7-10 days after elective knee arthroplasties.(1) These guidelines also recommend the extended use of prophylaxis, up to 35 days after the operation, in patients with a high risk of developing venous thromboembolism (VTE). Patients with a high risk of developing VTE include patients with active malignancies or previous VTE. Many orthopaedic surgeons believe that these recommendations are biased toward reducing deep venous thrombosis (DVT) at the expense of lower wound complications, which few surgeons are willing to accept. Furthermore, the incidence of pulmonary embolism is low regardless of the type of prophylaxis used.

We conducted a prospective observational study that looked at the incidence of DVT, as well as the time course of the development of DVT in the local population. Our study also aimed to examine the efficacy of the use of a short course of chemothromboprophylaxis $(<7$ days) in the prevention of venous thromboembolic events, to enable the objective evaluation of whether the ACCP recommendations are suitable for our local cohort of patients.

\section{METHODS}

This study was conducted in the Department of Orthopaedic Surgery, Singapore General Hospital, Singapore. The reconstructive surgeons in this department follow a standard departmental protocol. A total of 125 patients were enrolled into our study from February 2009 to December 2011. However, only 100 patients underwent both the early and one-month imaging; the remaining 25 patients did not turn up for their scheduled appointments for DVT imaging, and were thus not included in the final data analysis.

Of the 100 patients who completed both DVT imaging, 24 were male and 76 were female. The mean and median ages of the patients were 67 and 69 years, respectively, with the youngest patient aged 39 years, and the oldest, 85 years. The patients were predominantly standard or non-elevated risk patients, with no predisposition to thromboembolism. Standard risk patients were defined as those with no history of previous thromboembolic event, no blood clotting disorders, no history of trauma or fractures, no recent major surgery, no cancer

${ }^{1}$ Department of Orthopaedic Surgery, Singapore General Hospital, Singapore

Correspondence: Dr Low Mun Hon, Registrar, Department of Orthopaedic Surgery, Singapore General Hospital, Outram Road, Singapore 169608. mhlow1@gmail.com 
(current or previous), no spinal cord injury or other conditions that limit mobility, and no serious infections.

Prospective data of the patients, including their biodata, the types of chemothromboprophylaxis used, the period of initiation and duration of chemothromboprophylaxis use, as well as the rates of venous thromboembolic complication due to the use of chemothromboprophylaxis, was collected. The endpoint of the study was to determine the incidence of DVT in our local cohort. The incidence of DVT was determined using duplex ultrasonography, which was performed during the early postoperative days (postoperative Days 4-6), and one month after the operation. Such surveillance imaging was conducted on both the operated and non-operated lower limbs. DVT was diagnosed based on the criteria of visualisation of the thrombosis and the loss of compressibility of the vein.

Chi-square test was used to assess the categorical variables (e.g. sex and type of chemothromboprophylaxis used), while unpaired two-tailed $t$-test was used to assess the significance of the continuous variables (e.g. age and duration of chemothromboprophylaxis use).

\section{RESULTS}

Of the 125 patients recruited, only 100 patients completed the required postoperative imaging (one at postoperative Days 4-6 and another one month after the operation). Among these 100 patients, 52 had the operation done on their right knee, while 48 had the operation done on the contralateral side. The majority $(76 / 100)$ of the patients were female. The incidence of DVT post total knee arthroplasty (TKA) was $12 \%$ during postoperative Days $4-6$ and $9 \%$ one month after the operation (Table I). At both the early postoperative period (i.e. postoperative Days 4-6) and one month after the operation, only $2 \%$ of the DVT were symptomatic. The rest of the DVT detected were asymptomatic.

In the early postoperative period, $10 \%$ of the distal DVT and $1 \%$ of the proximal DVT correlated with the operated site. Only $1 \%$ of the distal DVT was detected on a nonoperated site. One month after TKA, 7\% of the distal DVT detected correlated with the site of operation, while $1 \%$ of the proximal DVT and $1 \%$ of the distal DVT were detected on the contralateral site of the operated limb.

Interestingly, 7 of the 9 cases of DVT detected one month after TKA were new cases that were not detected in the initial imaging conducted during postoperative Days 4-6. Only 2 of these 9 cases were detected previously. Of the 12 cases of DVT that were detected during the early postoperative period, $10(83 \%)$ resolved without causing pulmonary embolism.

When we compared the patients with and without DVT in the early postoperative period, we found no significant correlation between the incidence of DVT and age, gender, duration of chemothromboprophylaxis, type of chemothromboprophylaxis, and length of stay (Table II). There was also no significant correlation between the incidence of DVT and
Table I. Incidence of deep venous thrombosis (DVT) after total knee arthroplasty during postoperative Days 4-6 and one month after the operation $(n=100)$.

\begin{tabular}{lcc}
\hline Parameter & Days $\mathbf{4 - 6}$ & $\mathbf{1 ~} \mathbf{~ t h}$ \\
\hline Total DVT & 12 & 9 \\
Symptomatic DVT & 1 & \\
$\quad$ Proximal & 1 & 1 \\
$\quad$ Distal & & \\
Nonsymptomatic DVT & 0 & 0 \\
$\quad$ Proximal & 10 & 7 \\
$\quad$ Distal & 0 & 0 \\
\hline
\end{tabular}

Data is presented as percentage. PE: pulmonary embolism

the aforementioned factors when patients with and without DVT at one month after TKA were compared (Table III). There were no reported complications related to the use of chemothromboprophylaxis in our cohort. In other words, wound haematoma, bleeding and other complications related to bleeding were not an issue with the use of a short course of chemothromboprophylaxis.

\section{DISCUSSION}

Since DVT can result in life-threatening conditions such as pulmonary embolism, it is one of the most dreaded complications that can occur after TKA. Various factors, such as obesity, pregnancy, tobacco consumption, the presence of neoplasia, prolonged immobility, congestive heart failure, hypertension and diabetes mellitus, have been correlated with an increased risk of DVT. This condition, dubbed 'economy class syndrome', became headline news when studies concluded that DVT might occur in up to $10 \%$ of long-haul airline travellers, ${ }^{(2)}$ sparking public anxiety and inquisition into this significant and costly healthcare problem. DVT also poses a significant threat to a patient's general recovery and puts a significant strain on public health resources.

The orthopaedic community faces a tremendous challenge in finding the most effective strategy to prevent DVT, particularly in patients who have undergone total joint arthroplasty as they form a significant proportion of all orthopaedic patients. This daunting task is further aggravated with the numerous conflicting reports and recommendations present in the current literature. In an attempt to guide orthopaedic surgeons in establishing a consensus regarding the prevention of venous thromboembolic events after joint arthroplasty, the ACCP issued guidelines recommending the use of antithrombotic prophylaxis (e.g. low-molecular-weight heparin or warfarin, rivoroxaban, fondaparinux and apixaban) for a duration of at least 7-10 days after elective knee arthroplasties. ${ }^{(3)}$ These guidelines were developed based on an evaluation of the available literature and with prevention of distal DVT as the endpoint in the determination of the efficacy of various agents. However, the use of the prevention of distal DVT as an endpoint has been deemed inappropriate, as the prevention of such events has not been proven to be relevant 
Table II. Possible risk factors for deep venous thrombosis (DVT) during the early postoperative period (i.e. Days 4-6 after total knee arthroplasty) $(n=100)$.

\begin{tabular}{|c|c|c|c|}
\hline \multirow[t]{2}{*}{ Risk factor } & \multicolumn{2}{|c|}{ No. of patients } & \multirow[t]{2}{*}{ p-value } \\
\hline & With DVT & Without DVT & \\
\hline Mean age (yrs) & 63 & 68 & 0.089 \\
\hline Sex & & & 0.118 \\
\hline Male & 4 & 20 & \\
\hline Female & 8 & 68 & \\
\hline Duration of chemothromboprophylaxis use (mean days) & 5 & 4 & 0.563 \\
\hline Type of chemothromboprophylaxis* & & & 0.984 \\
\hline Clexane & 10 & 62 & \\
\hline Aspirin & 0 & 9 & \\
\hline Rivaroxaban & 1 & 14 & \\
\hline None & 0 & 1 & \\
\hline Length of stay (days) & 6 & 5 & 0.565 \\
\hline
\end{tabular}

${ }^{*}$ Total number of patients was 97 as 3 cases were indeterminate.

Table III. Possible risk factors for deep venous thrombosis $(D V T)$ at one month after total knee arthroplasty $(\mathrm{n}=100)$.

\begin{tabular}{|c|c|c|c|}
\hline \multirow[t]{2}{*}{ Risk factor } & \multicolumn{2}{|c|}{ No. of patients } & \multirow[t]{2}{*}{ p-value } \\
\hline & With DVT & Without DVT & \\
\hline Mean age (yrs) & 65 & 67 & 0.590 \\
\hline Sex & & & 0.614 \\
\hline Male & 1 & 23 & \\
\hline Female & 8 & 68 & \\
\hline Duration of chemothromboprophylaxis use (mean days) & 4 & 6 & 0.623 \\
\hline Type of chemothromboprophylaxis* & & & 0.879 \\
\hline Clexane & 8 & 64 & \\
\hline Aspirin & 0 & 3 & \\
\hline Rivaroxaban & 1 & 14 & \\
\hline None & 0 & 4 & \\
\hline Length of stay (days) & 5 & 7 & 0.625 \\
\hline
\end{tabular}

${ }^{*}$ Total number of patients was 94 as 6 cases were indeterminate.

in preventing life-threatening conditions such as pulmonary embolism. ${ }^{(4)}$

There are reports that thromboembolic events are rare in Asia. ${ }^{(5)}$ Studies have reported the incidence of proximal DVT in Asians to be only about $0.8 \%-1.6 \% .^{(6,7)}$ In a largescale prospective cohort study involving 128,934 patients, Klatsky et al also reported a lower incidence of DVT in Asians compared to other ethnic groups. ${ }^{(8)}$ This difference in the incidence of DVT has been postulated to be due to the lack of prothrombotic clotting factor polymorphisms among Asians. ${ }^{(9-11)}$ Furthermore, although studies conducted by Wang et $\mathrm{al}^{(10)}$ and Nathan et $\mathrm{al}^{\left({ }^{(11)}\right.}$ reported the incidence of all types of DVT to be as high as $64 \%$, the incidence of pulmonary embolism was still low in those studies.

In our study, we observed an incidence of proximal DVT of only $1 \%$ in both the early postoperative period and one month after TKA, with no clinical progression to pulmonary embolism. The overall DVT incidence was only $12 \%$ in the early postoperative period, and $9 \%$ one month after TKA. Follow-up imaging revealed that most of the DVT detected one month after TKA were new cases of DVT. Only $2 \%$ of the cases detected one month after TKA had images positive for DVT in the early postoperative period. This finding is in stark contrast to the findings of a study by Maynard et al, which revealed that $86 \%$ of the eventually-positive limbs were already positive within a day after surgery. ${ }^{(12)}$ They reported an incidence of total DVT of $47 \%$ during the early postoperative period and $54 \%$ during the late period. ${ }^{(12)}$

The incidence of major bleeding after TKA is $3 \%-6 \%$ in patients given chemothromboprophylactic agents..$^{(4,13-18)}$ It is noteworthy that the patients in our study, who were on a short course of chemothromboprophylaxis ( $<7$ days), did not have any significant major bleeding complications. The results of our study highlight the advantages of administering a short course of chemothromboprophylaxis ( $<7$ days), as the complication rate mimics that of patients not taking chemothromboprophylaxis. This would allay many orthopaedic surgeons' concern regarding the use of anticoagulation chemoprophylaxis and its associated risk of bleeding, blood transfusion and periprosthetic infection.

\section{REFERENCES}

1. Falck-Ytter $\mathrm{Y}$, Francis CW, Johanson NA, et al. Prevention of VTE in orthopedic surgery patients: Antithrombotic Therapy and Prevention of Thrombosis, 9th ed: American College of Chest Physicians Evidence-Based Clinical Practice Guidelines. Chest 2012; 141(2 suppl):e278S-325S.

2. Scurr JH, Machin SJ, Bailey-King S, et al. Frequency and prevention of symptomless deep-vein thrombosis in long haul flights: a randomized trial. Lancet 2001; 357:1485-9.

3. Geerts WH, Pineo GF, Heit JA, et al. Prevention of venous thromboembolism: 
the Seventh ACCP Conference on Antithrombotic and Thrombolytic Therapy. Chest 2004; 126(3 suppl):338S-400S.

4. Parvizi J, Azzam K, Rothman RH. Deep venous thrombosis prophylaxis for total joint arthroplasty: American Academy of Orthopaedic Surgeons guidelines. J Athroplasty 2008; 23(7 suppl):2-5.

5. Kim YH, Suh JS. Low incidence of deep vein thrombosis after cementless total hip replacement. J Bone Joint Surg Am 1988; 70:878-82.

6. Chin PL, Amin MS, Yang KY, Yeo SJ, Lo NN. Thromboembolic prophylaxis for total knee arthroplasty in Asian patients: a randomised controlled trial. J Orthop Surg (Hong Kong) 2009; 17:1-5.

7. Shen MC, Lin JS, Tsay W. High prevalence of antithrombin III, protein $\mathrm{C}$ and protein $\mathrm{S}$ deficiency, but no factor $\mathrm{V}$ Leiden mutation in venous thrombophilic Chinese patients in Taiwan. Thromb Res 1997; 87:377-85.

8. Klatsky AL, Armstrong MA, Poggi J. Risk of pulmonary embolism and/ or deep venous thrombosis in Asian-Americans. Am J Cardiol 2000; 85:1334-7.

9. Lin JS, Shen MC, TSay W. The mutation at position 20210 in the 3 -untranslated region of the prothrombin gene is extremely rare in Taiwanese Chinese patients with venous thrombophilia. Throb Haemost 1998; 800:343.

10. Wang CJ, Wang JW, Chen LM, et al. Deep vein thrombosis after total knee arthroplasty. J Formos Med Assoc 2000; 99:848-53.

11. Nathan S, Aleem MA, Thiagarajan $P$, Das De S. The incidence of proximal deep vein thrombosis folowing total knee arthroplasty in an Asian population: A Doppler ultrasound study. J Orthop Surg (Hong Kong) 2003; 11:184-9.

12. Maynard MJ, Sculco TP, Ghelman B. Progression and regression of deep vein thrombosis after total knee arthroplasty. Clin Orthop Relat Res 1991; 273:125-30.

13. Enyar JJ, Jones RJ, Low-dose warfarin for prevention of symptomatic thromboembolism after orthopaedic surgery. Ann Pharmacolother 2005; 39:1002-7.

14. Fitzgerald RH Jr, Spiro TE, Trowbridge AA, et al. Prevention of venous thromboembolic disease following primary total knee arthroplasty. J Bone Joint Surg Am 2001; 83-A:900-6.

15. Brookenthal KR, Freedman KB, Lotke PA, Fitzgerald $\mathrm{RH}$, Lonner JH. A meta-analysis of thromboembolic prophylaxis in total knee arthroplasty. J Arthroplasty 2001; 16:293-300.

16. Kim YH. The incidence of deep venous thrombosis after cementless and cemented knee replacement. J Bone Joint Surg Br 1990; 72:779-83.

17. Gallus AS, Salzman EW, Hirsh J. Prevention of venous thromboembolism. In: Colman RW, Hirsh J, Marder VJ, et al, eds. Haemostasis and thrombosis: basic principles and clinical practice, 3rd ed. Philadelphia:Lippincott, 1994: 1331-45.

18. Weinmann Ee, Salzman EW. Deep-vein thrombosis. N Engl J Med 1994; 331:1630-41. 